\title{
Effect of substrate roughness on photoluminescence spectra of silicon nanocrystals grown by off axis pulsed laser deposition
}

\author{
J. R. Rani ${ }^{a)}$ and V. P. Mahadevan Pillai \\ Department of Optoelectronics, University of Kerala, Kariavottom, Trivandrum, Kerala 695581, India \\ R. S. Ajimsha and M. K. Jayaraj \\ Optoelectronics Devices Laboratory, Department of Physics, Cochin University of Science and Technology, \\ Kochi, Kerala 682022, India \\ R. S. Jayasree \\ Department of Imaging Sciences and Interventional Radiology, Sree Chitra Tirunal Institute for Medical \\ Sciences and Technology, Trivandrum, Kerala 695011, India
}

(Received 22 September 2005; accepted 21 April 2006; published online 6 July 2006)

\begin{abstract}
Silicon nanoparticles were prepared by off axis pulsed laser deposition (PLD) technique. The optical properties of $\mathrm{Si}$ nanoparticles grown on quartz substrate have been characterized by optical absorption, photoluminescence, Raman, and transmission electron microscopy. TEM has demonstrated that the radius of nanocrystals decreases from 4 to $0.8 \mathrm{~nm}$ as the off axis target to substrate distance increases. A blueshift up to $4.2 \mathrm{eV}$ is observed in the optical absorption spectra of the Si quantum dots. The slope of $\log (h \nu)$ vs $\log (\alpha)$ graph shows that the optical transitions in $\mathrm{Si}$ quantum dots are allowed direct, allowed indirect, and direct forbidden transitions which depend on the nature of the substrate used for the growth of silicon. Relaxation of $k$ selection rule is observed in these samples. Photoluminescence (PL) emission consists of an intense broad emission extending over visible to ultraviolet region. The photoluminescence peak energy and intensity are found to be sensitive to the nature of substrate. Possible mechanism of optical absorption and PL origin are discussed in terms of quantum confinement luminescent center model. (C) 2006 American Institute of Physics. [DOI: 10.1063/1.2209432]
\end{abstract}

\section{INTRODUCTION}

Silicon $(\mathrm{Si})$ is the important semiconductor in the microelectronic industry. Recent reports on the intense visible photoluminescence from nanocrystalline silicon have attracted a great deal of attention due to the expectation of $\mathrm{Si}$ based optoelectronic devices. ${ }^{1-4}$ This discovery of visible photoluminescence (PL) from nanocrystalline Si has sparkled considerable debate regarding the mechanism for improved emission efficiency. ${ }^{5,6}$ Silicon based light emitting devices are highly desirable for the integration of optical signal and electronic data processing circuits on the same chip. Moreover the fabrication process is compatible with the present large scale integration technologies. ${ }^{7-9}$ Many researchers have investigated various methods for fabricating the $\mathrm{Si}$ nanoparticles as light emitters. Silicon quantum dots have excellent optical properties even in the short-wavelength region due to the quantum size effect. ${ }^{10}$ Silicon nanoparticles can be synthesized by several techniques such as ion implantation, ${ }^{11}$ electrochemical etching of silicon wafers, ${ }^{12,13}$ cosputtering, ${ }^{14}$ and pulsed laser deposition (PLD). ${ }^{15,16}$ The exact mechanism of light emission from Si nanoparticles is still a controversy, even though many investigations in this direction are in progress. The PL emission reported for silicon/silicon dioxide $\left(\mathrm{Si} / \mathrm{SiO}_{2}\right)$ systems consists of an intense emission peak in the near infrared, visible, and near ultraviolet (UV) regions. However, a broad spectral emission

${ }^{a)}$ Electronic mail: ranijnair@rediffmail.com is typically observed from Si nanocrystals. ${ }^{17}$ The PL emission from $\mathrm{Si} / \mathrm{SiO}_{2}$ systems consists of an intense broad peak at $1.95 \mathrm{eV}$, visible with the naked eye with a full width at half maximum (FWHM) of $0.3 \mathrm{eV} .{ }^{18-20}$ Wolkin et al. proposed that the trapping of an electron (or even an exciton) by silicon-oxygen double bonds produces localized levels in the band gap of nanocrystals. ${ }^{21}$ The interface between $\mathrm{Si}$ and $\mathrm{SiO}_{2}$ is not sharp which plays an attractive role in light emission. The PL is also been suggested due to defects with excess $\mathrm{Si}$ atoms in $\mathrm{SiO}_{2}$ films. ${ }^{22}$ The $\mathrm{Si}-\mathrm{O}$ vibrations at the interface makes a dominant path for the recombination of electron hole pairs in silicon nanocrystals embedded in $\mathrm{SiO}_{2}{ }^{23}$ Park et al. report that amorphous species Si used to confine $\mathrm{Si}$ nanocrystals can be responsible for light emission. ${ }^{24}$ Contradictory experimental results in the literature suggest that detailed study is needed to understand the light emission mechanism.

In the present investigation $\mathrm{Si}$ nanocrystals were prepared by off axis PLD. PLD has emerged as a promising technique because it is a cold wall processing which excites only a small area on the target by the focused laser beam. It is highly suited for the growth of quantum dots with high chemical purity and controlled stochiometry. In PLD, one can control size distribution of Si nanocrystals by varying the parameters such as target to substrate distance, laser fluence, background gas pressure, etc. The off axis PLD is an excellent method to produce nanoparticles. The laser ablation produces the plasma plume perpendicular to the target surface, which expanded towards substrate. Large $\mathrm{Si}$ crystals in $\mathrm{Si}$ 


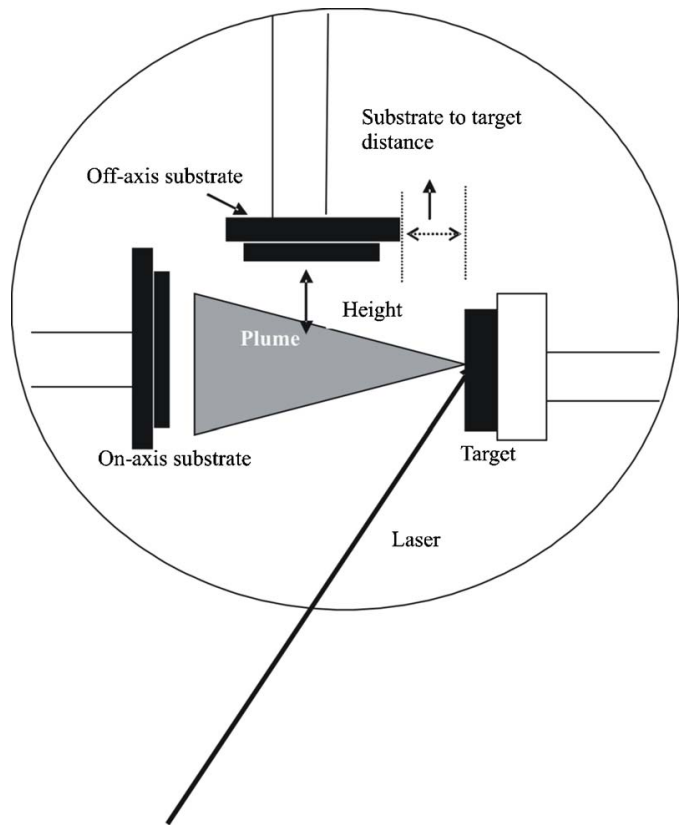

FIG. 1. Schematic diagram of off axis pulsed laser deposition.

plasma plume are more or less formed at the center of plume, smaller are distributed near plume edge. So off axis PLD is one of the most suitable choices due to its ability to control the size of the particles.

\section{EXPERIMENTAL DETAILS}

Silicon nanocrystals were synthesized by off axis pulsed laser ablation technique. The deposition was carried out using a $Q$-switched Nd:YAG (Yttrium aluminum garnet) laser with a fluence of $2 \mathrm{~J} / \mathrm{cm}^{2}$ at $532 \mathrm{~nm}$, having a pulse width of $9 \mathrm{~ns}$, and a repetition frequency of $10 \mathrm{~Hz}$. The target was rotated with constant speed to ensure uniform ablation. Chamber was evacuated to a base pressure of 1 $\times 10^{-6}$ mbar and high purity silicon pellet sintered at $1300{ }^{\circ} \mathrm{C}$ was used as a target material. Substrate was kept at target to substrate distance of $30 \mathrm{~mm}$ and at off axis heights of 5 and $10 \mathrm{~mm}$ with respect to laser plume (Fig. 1). Three types of nanofilms were synthesized. One set on a smooth quartz substrate for an ablation time of $18 \mathrm{~min}$, another one was coated on a rough quartz substrate for $18 \mathrm{~min}$, and the third set was a multilayer film of $\mathrm{Si}$ and $\mathrm{Al}_{2} \mathrm{O}_{3}$ with initial layer of alumina grown on the polished quartz substrate. The substrates were made rough by dipping the smooth quartz substrate in hydrogen fluoride (HF) solution for $15 \mathrm{~min}$.

For the preparation of multilayer, first layer of $\mathrm{Al}_{2} \mathrm{O}_{3}$ was grown on quartz substrate and after that, three layers of $\mathrm{Si}$ and three layers of $\mathrm{Al}_{2} \mathrm{O}_{3}$ were deposited alternatively. Ablation time for $\mathrm{Si}$ was 6 min and that for $\mathrm{Al}_{2} \mathrm{O}_{3}$ was $30 \mathrm{~s}$. In the multilayer structure, the growth may be in the form of isolated islands, which is capped by $\mathrm{Al}_{2} \mathrm{O}_{3}$ layer. The deposition of next layer of Si may result in the growth over the isolated islands. The multilayer film can be considered as $\mathrm{Si}$ dots evenly distributed over the substrates in the $\mathrm{Al}_{2} \mathrm{O}_{3}$ matrix.

In this paper we also report the studies of nanocrystalline $\mathrm{Si}$ particles using the transmission electron microscopy

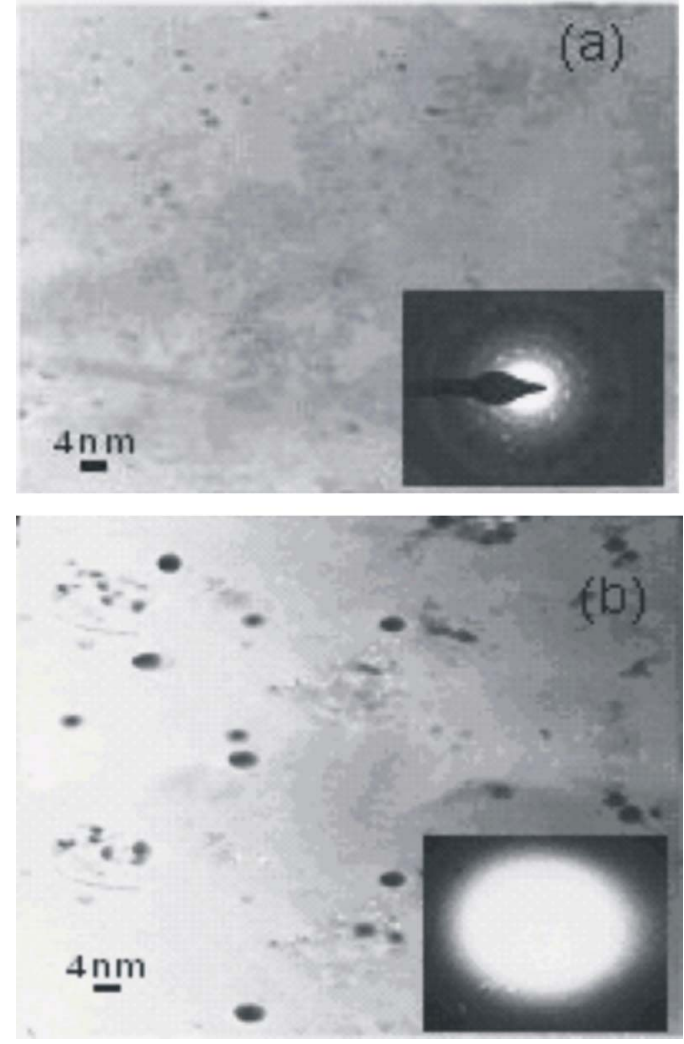

FIG. 2. TEM picture for Si nanoparticles grown by off axis PLD on $\mathrm{NaCl}$ crystal. Substrate to target distance is $30 \mathrm{~mm}$ and off axis heights; are (a) $10 \mathrm{~mm}$ and (b) $5 \mathrm{~mm}$. Inset shows the selective area diffraction pattern.

(TEM). The Si nanoparticles were deposited on $\mathrm{NaCl}$ crystals by off axis PLD at a target to substrate distance of $30 \mathrm{~mm}$, keeping the heights at 5 and $10 \mathrm{~mm}$ for a deposition time of $18 \mathrm{~min}$. The TEM shows a variation of particle size as a function of off axis height. The nanostructure of films was examined by a Hitachi H-600 TEM operated at $500 \mathrm{kV}$. Raman spectra of samples were measured with Raman spectrometer (Nicolet Thermo) at room temperature using $532 \mathrm{~nm}$ line of Ar-ion laser for excitation. Optical absorption spectra were recorded using an UV-VIS-NIR spectrophotometer (Hitachi U 3410) in the spectral range of 200-800 nm. The PL measurements were recorded by Jobin Yvon spectrofluorometer (Fluorolog III). The crystal structure was examined using x-ray diffractometer (Rigaku) with $\mathrm{Cu} K \alpha(\lambda$ $=1.5418 \AA$ ) radiation.

\section{RESULTS AND DISCUSSION}

\section{A. TEM analysis}

The nanostructures of as deposited films were examined by TEM observations. Figures 2(a) and 2(b) show TEM images for Si quantum dots prepared by an off axis PLD at a substrate to target distance of $30 \mathrm{~mm}$ and off axis heights of 10 and $5 \mathrm{~mm}$ respectively. The on axis substrate to target distance was kept the same in both cases. The quantum dots are uniformly dispersed in films and majority of dots are having a spherical shape and some of them are ellipsoidal. For a substrate to target distance of $10 \mathrm{~mm}$ the average particle size is found to be $0.8 \mathrm{~nm}$ whereas for a distance of 

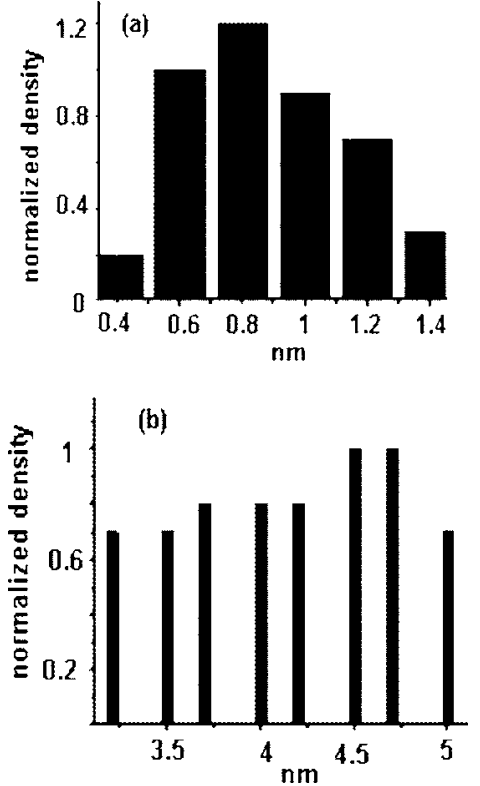

FIG. 3. Silicon size distribution obtained by TEM analysis for samples deposited at substrate to target distances of (a) $10 \mathrm{~mm}$ and (b) $5 \mathrm{~mm}$. Height is $30 \mathrm{~mm}$.

$5 \mathrm{~mm}$ the crystallites have a size around $4 \mathrm{~nm}$. TEM micrograph shows that smaller mean size $\mathrm{Si}$ nanocrystals are formed when the off axis target to substrate distance increases. This technique gives a better control on the size of nanoparticles. Figure 3 shows size distribution of Si nanoparticles grown by off axis PLD for off axis substrate to target distances of 5 and $10 \mathrm{~mm}$. During the ablation of $\mathrm{Si}$, large crystals are formed at the center of the plasma plume, while smaller particles are formed near the edge of the plasma. The larger nanocrystals are formed on the substrate kept at the center of the plume. In the present study, all the samples were deposited by keeping the substrate in the off axis geometry. The variation of size distribution of Si nanocrystals with substrate to target distance may be due to the collision of ejected species. An increase in substrate to target distance will result in increased collisions between the ejected species and heavier particles may not reach the substrate. At small substrate to target distance, the solidified droplets of Si expelled from the target may get deposited on substrate along with various species and result in bigger crystallites. However, the position of substrates in the present investigation was such that bigger droplets if any expelled from the target fall down by the gravity.

The instability of diamond structure in the $\mathrm{Si}$ particle with free surface having less than $3 \mathrm{~nm}$ in diameter has been predicted. ${ }^{25}$ The selective area diffraction pattern shows the cubic crystal structure for the films deposited at an off axis height of $10 \mathrm{~mm}$.

\section{B. Raman spectra}

Raman spectrum of the crystalline silicon and the film coated on smooth quartz substrate is shown in Fig. 4. The films coated on smooth quartz substrate show a peak at $502 \mathrm{~cm}^{-1}$. The shifting of this band to lower wave numbers and enhancement of intensity of this mode may be due to

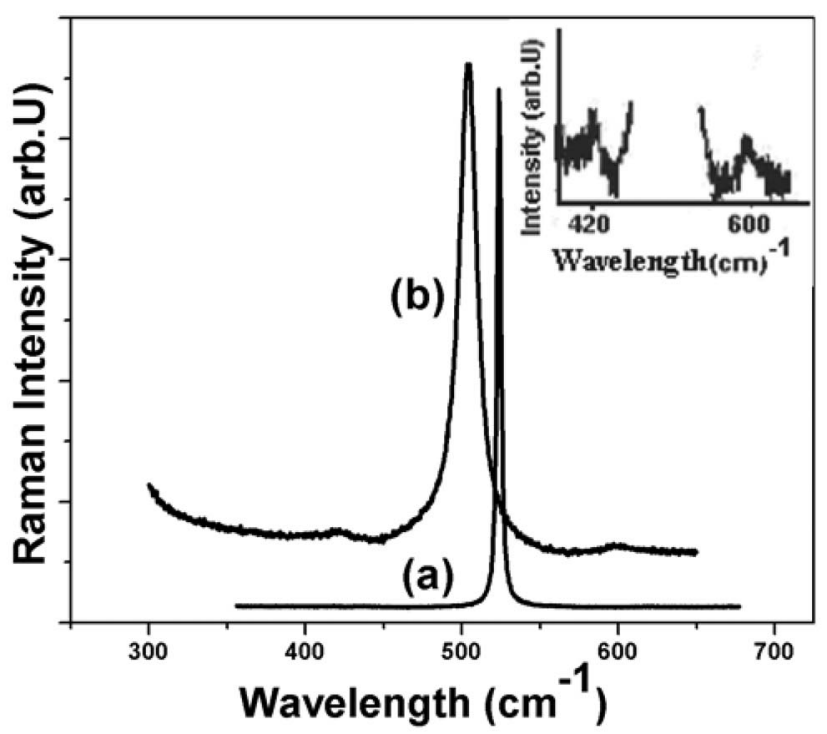

FIG. 4. Recorded Raman spectrum of silicon (a) for the bulk crystalline Si and (b) Si coated on smooth quartz substrate. Inset shows magnified portion of (b).

quantum confinement effect. The shift of Raman peaks to lower wave numbers compared to the Raman peak of crystalline $\mathrm{Si}$ can be attributed to phonon confinement effects, while the larger FWHM can be correlated to size distribution of the crystals. ${ }^{26}$ Two weak broad bands observed at 420 and $599 \mathrm{~cm}^{-1}$ in the Raman spectrum of the film coated on smooth quartz substrate are shown in the inset of Fig. 4. These peaks are observed only in the films coated on smooth quartz substrate, suggesting the significance of the relaxation of crystal momentum conservation rule in silicon nanoparticles. ${ }^{27-29}$ The optical transmission studies on this film show the indirect forbidden transition with a band gap of $1.88 \mathrm{eV}$ (Fig. 7). In the case of multilayer structure and samples coated on rough quartz substrate (Fig. 5) a fluctuation in stress in crystalline regions of Si may cause the broadening of Raman peak than that of bulk Si. Raman spectra are strongly affected by scattering from the substrate.

Figure 6 shows the x-ray diffraction (XRD) pattern of $\mathrm{Si}$ nanocrystals grown by off axis PLD. The diffraction pattern shows the (111) peak of the silicon along with two peaks at $14.08^{\circ}$ and $16.2^{\circ}$ due to the presence of silicon dioxide 1 -aminoadamantane deca-dodecasil-3R. The peak at $16.2^{\circ}$ corresponds to (107) plane and the peak at $14.08^{\circ}$ corresponds to (113) plane of silicon dioxide. ${ }^{30}$ The sharp peak

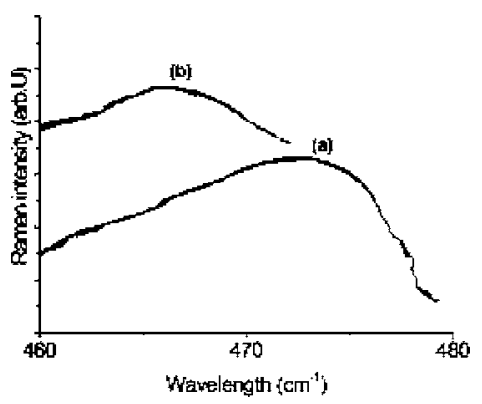

FIG. 5. Raman spectra for films (a) coated on rough quartz substrate and (b) multilayer structure. 


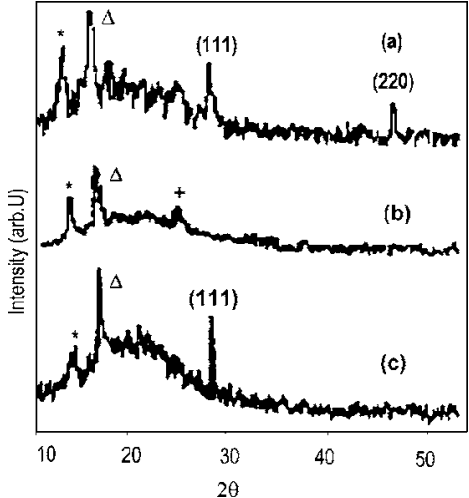

FIG. 6. The XRD pattern of silicon nanoparticles coated on (a) rough quartz substrate, (b) multilayer structure, and (c) smooth quartz substrate. (*) (113) plane of silicon dioxide 1-aminoadamantane deca-dodecasil-3R, $(\triangle)(107)$ plane of silicon dioxide 1-aminoadamantane deca-dodecasil-3R, and $(+)$ peak of $\mathrm{Al}_{2} \mathrm{O}_{3}$.

observed in the film deposited on smooth quartz corresponds to (111) plane of Si with $d$ value of $3.125 \AA$ (diamond structure). Apart from prominent (111) peaks, (220) peaks are also present in quantum dots coated on rough substrate. These peaks are due to random orientation of nanocrystals on rough substrate. The growth direction is highly probable along the (111) planes since they have highest atomic density and lowest surface energy. In the multilayer structure the peak at $25.27^{\circ}$ with $d$ value of $3.5 \AA$ corresponds to $\mathrm{Al}_{2} \mathrm{O}_{3}$. XRD pattern suggests that there is formation of silicon dioxide in all the films. The lattice constant of Si quantum dots is found to be $5.43 \AA$, is confirming the cubic structure of Si observed from the selective area electron diffraction.

\section{Optical absorption spectra}

The optical band gap $E_{g}$ can be estimated from the following relation which is known as the Tauc plot: ${ }^{31}$

$$
\alpha h \nu=A\left(h \nu-E_{g}\right)^{n},
$$

where $E_{g}$ is the band gap corresponding to a particular transition occurring in the film, $A$ is a constant, $\nu$, is the transition frequency and the exponent $n$ characterizes the nature of band transition. $n=1 / 2$ and $3 / 2$ corresponds to direct allowed and direct forbidden transitions, and $n=2$ and 3 corresponds to indirect allowed and indirect forbidden transitions, respectively. ${ }^{32,33}$ The band gap can be obtained from extrapolation of the straight-line portion of the $(\alpha h \nu)^{1 / n}$ vs $h \nu$ plot to $h \nu=0$. From the inset of Fig. 7, it is observed that for the $\mathrm{Si}$ and $\mathrm{Al}_{2} \mathrm{O}_{3}$ multilayer structure the best straight line has obtained for $n$ nearly equal to $3 / 2$ which is expected for direct forbidden transition and the corresponding band gap value is about $3.7 \mathrm{eV}$. But for a film on rough substrate $n$ is found to be close to $1 / 2$, which corresponds to a direct transition and the band gap is $4.2 \mathrm{eV}$. The film coated on smooth quartz substrate value of $n$ is found to be 3.59 , expected for indirect forbidden transition, and band gap is around $1.85 \mathrm{eV}$. For all samples, absorption threshold of silicon crystallites is higher than that of bulk $\mathrm{Si}$ and shifts towards high-energy side with decreasing average size of crystallites. In multilayer structure the layer thickness that is of the order
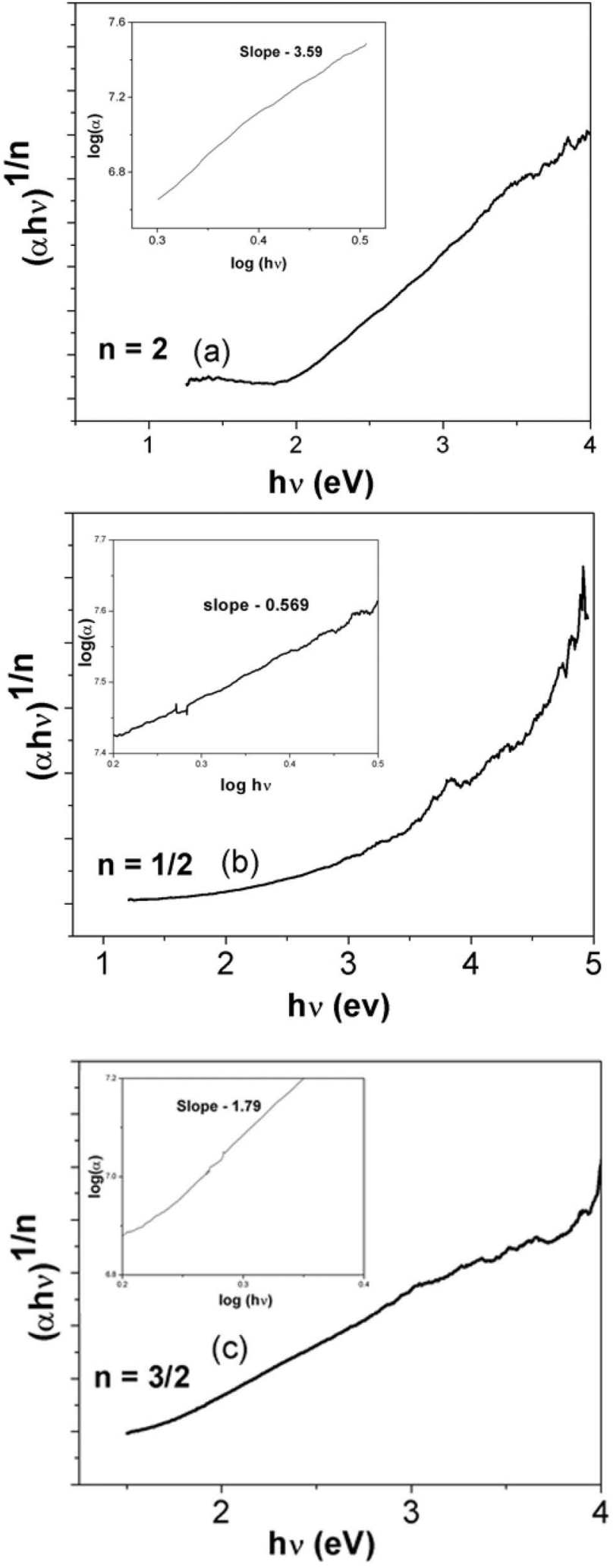

FIG. 7. Plot of $(\alpha h \nu)^{n}$ against $h \nu$ for silicon quantum dots coated on (a) smooth quartz substrate, (b) multilayer structure, and (c) rough quartz substrate. Inset shows variation of $\log (h \nu)$ with $\log (\alpha)$.

of the unit cell dimensions can result in a direct or quasidirect band gap structure. ${ }^{34}$

The polar nature of $\mathrm{Si}-\mathrm{O}$ bond causes the trapping of exciton adjacent to the interface of $\mathrm{Si}-\mathrm{SiO}_{2} \cdot{ }^{35}$ The spatial 


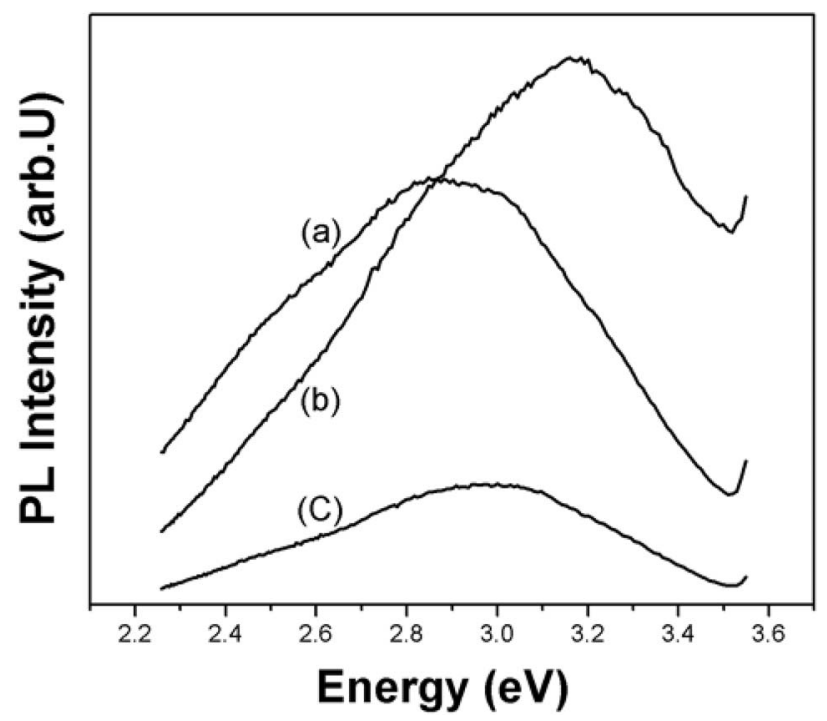

FIG. 8. Photoluminescence spectra of $\mathrm{Si}$ nanocrystals coated on (a) multilayer structure, (b) rough quartz substrate, and (c) smooth quartz substrate.

confinement of excitons in small nanoparticles leads to the spread of $k$ in reciprocal lattice and may violate the $k$ selection rule. This enhances the probability of direct optical transition. ${ }^{36,37}$ In our case in the samples coated on HF etched quartz substrate (rough substrate), there can be a strong chance for the formation of $\mathrm{Si}-\mathrm{O}-\mathrm{H}$ bond and its polar nature may cause the confinement of excitons in nanoparticles and may result in a direct optical transition.

\section{Photoluminescence studies}

Figure 8 shows the room temperature PL of as deposited $\mathrm{Si}$ nanocrystals. All the samples are characterized by strong PL emission in near UV-visible region. The PL peak emissions are observed at $3.19,3$, and $2.8 \mathrm{eV}$ for films coated on rough quartz substrate, smooth quartz substrate, and multilayer structures, respectively. However, a remarkable fact is that the difference between band gap obtained from the optical absorption spectra and PL emission is approximately constant for all samples and has a value of about $1 \mathrm{eV}$. The energy difference between absorption threshold and PL peak may be due to the relaxation of photoexcited carriers into the surface states. PL emission intensity of films deposited on rough quartz substrate and multilayer structure is higher than that of Si nanoparticles on smooth substrate.

In Brodsly's model, PL is believed to be the result of recombination of e-h pairs in $\mathrm{Si}$ well. ${ }^{38}$ The suggestion that luminescence is likely to originate from contaminations or defective silicon oxides has been voiced in a number of papers. ${ }^{39,40} \mathrm{PL}$ emission process in a nanoscale Si/silicon oxide system with an average size of about $3 \mathrm{~nm}$ took place at the interface between silicon nanocrystals and $\mathrm{SiO}_{2}$ matrix. ${ }^{41}$ Some authors put forward quantum confinement luminescent center (QCLC) model in which photoexcitation occurs in nanoparticles and photoemission occurs in luminescent centers (LCs) in the surrounding Si oxide layer and radiatively recombine there. ${ }^{42}$ The reactive model proposed by Iwayama et al. also concluded that quantum confinement effects play a role in photoabsorption and nanocrystalline $\mathrm{Si} / \mathrm{SiO}_{2}$ interface plays role in luminescence. ${ }^{43}$ Our results suggest that more than one type of mechanism is needed to interpret the $\mathrm{PL}$ from nanoscale $\mathrm{Si} / \mathrm{Si}$ oxide systems.

In the present case the films were produced by depositing the nanoparticle $\mathrm{Si}$ in an oxygen-free environment, oxygen molecules penetrate into films, and $\mathrm{SiO}_{2}$ layer is formed on the surface of individual silicon nanoparticles when the films are exposed to air. Oxidation introduces $\mathrm{Si}-\mathrm{SiO}_{2}$ interface or defects in oxide layer. ${ }^{44} \mathrm{Si}-\mathrm{SiO}_{2}$ composites are essential factors to understand their emission mechanism. Oxidation helps the formation of radiative states in an interfacial region between the silicon oxide layer and silicon core. Oxygen atoms critically modify the electronic structure of $\mathrm{Si}$ nanostructures. The interfacial region is assumed to have energy gap wider than that of Si core. Also the interfacial band gap depends on whether the surface termination is hydrogen or oxygen. $\mathrm{Si}=\mathrm{O}$ is more likely to be formed in the interfacial region for films coated on smooth quartz substrate and multilayers whereas there may be a strong chance for the formation of $\mathrm{Si}-\mathrm{O}-\mathrm{H}$ in the interfacial region for the films coated on HF etched quartz substrate. These bonds stabilize the interface, and terminate two dangling bonds. Si nanoclusters terminated with hydrogen have larger band gaps than the corresponding cluster with double-bonded oxygen. Also wave function localization of some near-band-edge states on the interface of $\mathrm{Si}=\mathrm{O}$ bonds is stronger than that of $\mathrm{Si}-\mathrm{O}-\mathrm{H}$ bonds which makes the interface of $\mathrm{Si} / \mathrm{SiO}_{2}$ sharper for films coated on smooth quartz substrate and multilayers. So after the absorption of photon energy, the carriers are trapped in the $\mathrm{Si}=\mathrm{O}$ bond and $\mathrm{Si}-\mathrm{O}-\mathrm{H}$ bonds and the PL results due to the transition from this level to ground state. Reports show that ground state energy varies with energy level of valence band. In the present studies for films coated on rough quartz substrate, direct transition is expected and there may be a chance for the change of valence band edge. The higherenergy level of $\mathrm{Si}-\mathrm{O}-\mathrm{H}$ bonds and shift of valence band edge may cause a blueshift for films coated on rough quartz substrate.

Thus it is interesting to note that this difference occurs despite the fact that nanocrystal preparation conditions are identical, the only difference between the two films is the substrate roughness. The substrate effect plays a role in oxidation of the samples. Structural characterization of silicon nanocrystals and study of their local environment are fundamental to understand the light emission process. This experimental work confirms the significant role of substrate in oxidation of samples and formation of interfacial regions.

\section{CONCLUSIONS}

Silicon quantum dots with an average size of $1 \mathrm{~nm}$ were synthesized by off axis pulsed laser deposition. TEM analysis demonstrates that mean size of silicon nanocrystals has a direct dependence on the off axis height. The band gap energies of quantum dots are observed to be $1.85 \mathrm{eV}$ for dots coated on smooth quartz substrate, around $3.7 \mathrm{eV}$ for multilayer structure, and $4.2 \mathrm{eV}$ for the dots grown on rough quartz substrate. The blueshift in the absorption threshold is 
due to quantum confinement effects in silicon nanocrystals. From the absorption spectra it has been observed that the nature of transition depends on the nature of substrate and the structure of multilayer. The shift in Raman band to lower wave numbers and enhancement of its intensity are attributed to quantum confinement effect. The synthesized films exhibit PL peak in the UV-visible region. The PL emission peak and intensity are dependent on the nature of substrate. It is concluded that luminescence does not originate from localized states in gap but from extended states.

\section{ACKNOWLEDGMENTS}

The authors would like to acknowledge Kerala State Council for Science Technology and Environment (KSCSTE) for the financial support.

${ }^{1}$ T. Canham, Appl. Phys. Lett. 57, 1046 (1990).

${ }^{2}$ D. Kovalev and H. Heckler, Phys. Status Solidi B 215, 871 (1999).

${ }^{3}$ H. Takagi, H. Ogawa, Y. Yamazaki, A. Ishizaki, and T. Nakagiri, Appl. Phys. Lett. 56, 2379 (1990).

${ }^{4}$ Y. T. Tan, T. Kamiya, Z. A. K. Durrani, and H. Ahmed, J. Appl. Phys. 94 33 (2003)

${ }^{5}$ M. W. M. Van Cleef, R. E. I. Schropp, and F. A. Rubinelli, Appl. Phys. Lett. 73, 2609 (1998).

${ }^{6}$ I. C. Cheng and S. Wagner, Appl. Phys. Lett. 80, 440 (2002).

${ }^{7}$ H. Tamura, M. Ruckschloss, T. Wirschem, and S. Veprek, Appl. Phys. Lett. 65, 1537 (1994).

${ }^{8}$ L. S. Liao, X. M. Bao, X. Q. Zheng, N. S. Li, and N. B. Min, Appl. Phys. Lett. 68, 850 (1996).

${ }^{9}$ G. G. Siu, X. L. Wu, Y. Gu, and X. M. Bao, Appl. Phys. Lett. 74, 1812 (1999).

${ }^{10}$ K. Yoshihiko, U. Hiroshi, M. Yasuaki, and M. Yoshihito, Appl. Phys. Lett. 61, 2187 (1992).

${ }^{11}$ T. S. Iwayama, S. Nakao, and K. Saitoh, Appl. Phys. Lett. 65, 1814 (1994).

${ }^{12}$ K. H. Jung, S. Shih, T. Y. Hsieh, D. L. Kwong, and T. L. Lin, Appl. Phys. Lett. 59, 3264 (1991).

${ }^{13}$ G. Belomoin et al., Appl. Phys. Lett. 80, 841 (2002).

${ }^{14}$ R. K. Soni, L. F. Fonseca, O. Resto, M. Buzaianu, and S. Z. Weisz, J. Lumin. 83, 187 (1999).

${ }^{15}$ H. C. Le, R. W. Dreyfus, W. Marine, M. Sentis, and I. A. Movtchan, Appl. Surf. Sci. 96, 164 (1996).

${ }^{16}$ T. Makino, Y. Yamada, N. Suzuki, T. Yoshida, and S. Onari, J. Appl. Phys. 90, 5075 (2001).
${ }^{17}$ S. Furukawa and T. Miyasato, Jpn. J. Appl. Phys., Part 2 27, L2207 (1988).

${ }^{18}$ H. Nishikawa, E. Watanabe, D. Ito, Y. Sakurai, K. Nagasawa, and Y. Ohki, J. Appl. Phys. 80, 3513 (1996).

${ }^{19}$ H. Nishikawa et al., J. Non-Cryst. Solids 222, 221 (1997).

${ }^{20}$ Y. Sakurai, K. Nagasawa, H. Nishikawa, and Y. Ohki, J. Appl. Phys. 86, 370 (1999).

${ }^{21}$ M. V. Wolkin, J. Jorne, P. M. Fauchet, G. Allan, and C. Delerue, Phys. Rev. Lett. 82, 197 (1999).

${ }^{22}$ T. Makimura, Y. Kunni, N. Ono, and K. M. Urakanni, Appl. Surf. Sci. 127, 388 (1998).

${ }^{23}$ B. Garrido, M. Lopez, O. Gonzalez, A. P. Rodriguez, J. R. Morante, and C. Bonafos, Appl. Phys. Lett. 77, 3143 (2000).

${ }^{24}$ N. M. Park, C. J. Choi, T. Y. Seong, and S. J. Park, Phys. Rev. Lett. 86, 1355 (2001)

${ }^{25}$ U. Rothlisberger, W. Andreoni, and M. Parrinello, Phys. Rev. Lett. 72, 665 (1994).

${ }^{26}$ T. I. Campbell and F. Faucchet, Solid State Commun. 58, 739 (1981).

${ }^{27}$ J. Liu, J. Niu, D. Yang, M. Yan, and J. Sha, Physica E (Amsterdam) 23, 221 (2004).

${ }^{28}$ Z. Iqbal and S. Veperk, J. Phys. C 15, 377 (1982).

${ }^{29}$ Z. Sui, P. P. Leong, L. P. Herman, G. S. Higasni, and X. Ternkin, Appl. Phys. Lett. 60, 2085 (1992).

${ }^{30}$ Joint Committee on Powder Diffraction Standards, Powder Diffraction File, Card No. 9-185 (1967).

${ }^{31}$ J. Tauc, Amorphous and Liquid Semiconductors (Plenum, London, 1974), p. 159.

${ }^{32}$ A. Goswami, Thin Film Fundamentals (New Age International (p) Limited, New Delhi, India, 1996).

${ }^{33}$ J. I. Pankove, Optical Process in Semiconductors (Prentice-Hall, Englewood Cliffs, NJ, 1971), p. 34.

${ }^{34}$ U. Gnutzman and K. Clausecker, Appl. Phys. 3, 9 (1974).

${ }^{35}$ B. G. Fernandez, M. Lopez, C. Garcia, A. P. Rodriguez, C. Bonafos, M. Carrada, A. Claverie, and J. R. Morante, J. Appl. Phys. 91, 798 (2002).

${ }^{36}$ N. Zhao and O. Schoenfeld, Phys. Rev. B 50, 18654 (1994).

${ }^{37}$ T. Takagahara and K. Takeda, Phys. Rev. B 46, 15578 (1992).

${ }^{38}$ M. H. Brodsly, Solid State Commun. 36, 55 (1980).

${ }^{39}$ G. Cullis, L. T. Canham, and P. D. J. Calcott, J. Appl. Phys. 82, 909 (1997).

${ }^{40}$ H. Xie, W. L. Wilson, F. M. Roes, J. A. Mucha, E. A. Fitzgerald, J. M. Macaully, and T. D. Harris, J. Appl. Phys. 71, 2403 (1992).

${ }^{41}$ L. Pavesi, L. D. Negro, C. Nazzoleni, G. Franzo, and P. Priolo, Nature (London) 408, 440 (2000).

${ }^{42}$ G. G. Qin and Y. Q. Jia, Solid State Commun. 86, 559 (1993)

${ }^{43}$ T. S. Iwayama, N. Kurumodo, D. E. Hole, and P. D. Townsend, J. Appl. Phys. 83, 6018 (1999).

${ }^{44}$ Y. Kanemitsu, T. O. Gawa, K. Shiraishi, and K. Takada, Phys. Rev. B 48, 4883 (1993). 